\title{
Estudios del lenguaje desde una perspectiva glotopolítica*
}

\author{
Language Studies from a Glottopolitical Perspective \\ Estudos da linguagem sob uma perspectiva glotopolítica
}

\author{
Juliana Angélica Molina Ríos ${ }^{\text {a }}$ \\ Pontificia Universidad Javeriana, Colombia \\ juliana.molina@javeriana.edu.co \\ ORCID: http://orcid.org/0000-0002-5858-3857
}

\author{
DOI: https://doi.org/10.11144/Javeriana.syp38-74.elpg \\ Redalyc: http://www.redalyc.org/articulo.oa $? \mathrm{id}=86059657009$
}

Fecha de recepción: 13 Febrero 2018

Fecha de aprobación: 10 Diciembre 2018

Fecha de publicación: 18 Junio 2019

\section{Resumen:}

El artículo presenta algunas reflexiones derivadas del proyecto de investigación doctoral relacionado con los "Ideologemas sobre los procesos de formación de excombatientes después de la firma del Acuerdo de Paz en Colombia". Se presentan los temas y problemas de estudio que se han abordado desde la glotopolítica, especialmente desde los ideologemas. La glotopolítica, como campo de conocimiento que se vincula a la sociolingüística, se pregunta por las prácticas lingüísticas que legitiman, reproducen y transforman las relaciones sociales y las estructuras de poder. En el presente escrito, se desarrollan tres apartados: a) las representaciones ideológicas del lenguaje, b) la función política de la lengua en diccionarios, gramáticas y manuales de texto y c) las políticas lingüisticas: normatividad y regulación lingüísticas, los cuales dan cuenta del creciente interés por comprender el lenguaje y la lengua articulados a la política.

Palabras clave: glotopolítica, política, representaciones, enseñanza de lengua, español.

\section{Abstract:}

This article provides some reflections stemming from the doctorate research project concerning the "ideologhemes on the formation processes for former combatants after signing the Peace Agreement in Colombia". This article introduces the topics and study problems dealt with by the glottopolitics, particularly from the ideologhemes. Glottopolitics as a field of knowledge linked to the sociolinguistics inquires into the linguistic practices legitimating, reproducing and transforming the social relationships and the power structures. This paper develops in three parts: a) the ideological representations of language; $b$ ) the political function of the language in dictionaries, grammar books and text manuals; and c) the linguistic policies: linguistic norms and regulations accounting for the growing interest in understanding both the language and the country language articulated with then politics. Keywords: glottopolitics, politics, representations, language learning, Spanish.

\section{Resumo:}

O artigo apresenta reflexões derivadas do projeto de pesquisa doutoral relacionado aos "Ideologemas sobre os processos de formação de excombatentes após a assinatura do Acordo de Paz na Colômbia”. Questões e problemas de estudo abordados desde a glotopolítica, em especial desde os ideologemas são apresentados. A glotopolítica, como campo de conhecimento vinculado à sociolinguística, pergunta pelas práticas linguísticas que legitimam, reproduzem e transformam as relações sociais e as estruturas de poder. No atual escrito, desenvolvem-se três apartados: a) as representações ideológicas da linguagem, b) a função política da língua em dicionários, gramáticas e manuais de texto e c) as políticas linguísticas: normatividade e regulamentação linguística, que dão conta do crescente interesse por compreender a linguagem e a língua articuladas à política.

Palavras-chave: glotopolítica, política, representações, ensino da língua, espanhol.

Notas de autor:

a Autora de correspondencia. Correo electrónico: juliana.molina@javeriana.edu.co 


\section{Introducción}

El presente artículo da cuenta de los estudios relacionados con el campo de la glotopolítica, el cual se ocupa de estudiar las distintas formas en que las prácticas lingüísticas, las acciones sobre las lenguas y las relaciones entre estas últimas participan en la conformación, reproducción o transformación de las relaciones sociales y de las estructuras de poder (López, 2016, p. 15). Es decir, la glotopolítica se pregunta por las prácticas lingüísticas que legitiman, reproducen y transforman las formas y luchas del reconocimiento del otro, articuladas a las estructuras de poder.

En este contexto, situar la lengua en la acción colectiva implica tener en cuenta categorías centrales para su análisis como lo son: el poder, la autoridad y la legitimidad. En palabras de Arnoux y Bein (2015), las lenguas se valorizan como símbolos de identidad cuando legitiman o deslegitiman hablantes y prácticas sociales, dado que producen y reproducen las diferencias, ubicando a cada uno en el lugar social que supuestamente le corresponde (p. 13). Así, la lengua refleja un orden social, pero también da forma a lo social y a la interacción entre el individuo y la sociedad.

De allí que el interés por comprender la lengua desde la perspectiva glotopolítica se dé en varios sentidos: el primero tiene que ver con el reconocimiento de que la lengua se modifica en la interacción social y esta, a su vez, se transforma por la influencia de la primera; el segundo está relacionado con el hecho de que las prácticas lingüísticas están asociadas con la política, específicamente, con las formas de poder; por último, el tercero reconoce que en la lengua se encuentran ancladas las prácticas de interacción social. En síntesis, se estudia la lengua como práctica sociopolítica.

Considerando lo anterior, se presentan los temas y problemas relacionados con la glotopolítica. Para ello, se desarrollan tres apartados: a) las representaciones ideológicas del lenguaje (ideologías lingüísticas resultado de acontecimientos históricos), b) la función política de la lengua (diccionarios, gramáticas y manuales de texto) y c) las políticas lingüísticas (normatividad y regulación lingüísticas).

\section{Temas y problemas de la glotopolítica: configuraciones de su estudio}

Los temas y problemas en los que se ha centrado la glotopolítica están relacionados con el interés por comprender los hechos y las acciones del lenguaje que se desprenden de determinadas situaciones de una sociedad o grupo social, ya sean estas de carácter político, social, cultural o económico, y que se configuran a través de la lengua, el habla y el discurso. En otras palabras, "la glotopolítica designa las diversas formas en que una sociedad actúa sobre el lenguaje [...]. Es un término necesario para englobar todos los hechos del lenguaje en los que la acción de la sociedad reviste la forma de lo político" (Guespin y Marcellesi, 1986 citados por Lauria, 2009, p. 1). Así, en la configuración de estos hechos del lenguaje, se pueden identificar tres grandes campos de reflexión, que a continuación se explicitan.

\section{Las representaciones del lenguaje: ideologías lingüísticas resultado de acontecimientos históricos}

La lengua que hablamos y escribimos es portadora del recorrido histórico que la trajo como variedad de uso hasta nuestros dias. María López, 2016

Dentro del campo de la glotopolítica, un tema de interés central ha sido el estudio de las representaciones del lenguaje a partir de las ideologías lingüísticas. En este marco, Del Valle (2014) ha sido uno de los principales investigadores que se ha enfocado en la comprensión de la representación del lenguaje y la glotopolítica. El autor hace un recorrido histórico de la glotopolítica, donde destaca tres corrientes: la primera 
aparece después de la Segunda Guerra Mundial, "se procede a crear un aparato teórico y metodológico que permita describir el perfil sociolingüístico de una comunidad para, a la vez, orientar la toma de decisiones políticas que incidan o afecten tal perfil” (p. 89), es decir, se proponen formas de organización lingüísticas acordes con determinadas comunidades. Así mismo, en esta primera corriente, surgen discusiones en torno a las categorías de diglosia y esquizoglosia, ordenamiento lingüístico, valor de las lenguas, identidad y la naturaleza de la estandarización.

La segunda corriente que se origina en la década de los 60 toma una posición crítica frente a la anterior y se opone a la misma en la medida en que la primera "propone modelos de organización lingüística que perpetúan las jerarquías propias del colonialismo" (Del Valle, 2014, p. 89). Desde esta posición, se cuestiona el concepto de bilingüismo armónico, lo que apunta a que el contacto lingüístico sea conflictivo.

La tercera corriente, que toma lugar a finales del siglo XX, "está ligada a los procesos políticos y económicos de integración regional, a la formación de redes de interacción transnacionales y a los flujos de capital, gente e información típicamente asociados con la globalización. Se negocian, en esta fase, tensiones entre el valor cultural, político y económico de las lenguas en los mercados lingüísticos” (Del Valle, 2014, p. 90). Sin embargo, en esta corriente, también se evidencian las reivindicaciones de las comunidades que representan minorías lingüísticas y entidades que se encargan de los campos de enseñanza de lenguas, traducción, entre otros.

Desde este contexto, para Del Valle (2014), el punto en común que caracteriza las anteriores corrientes es el modo en que se piensa o identifica el lenguaje, es decir, este es visto como "un ámbito de la vida social susceptible de ser objeto de la acción política" (p. 90). De allí que se plantea la relación entre lenguaje y política, en la cual intervienen la distribución de poder ${ }^{1}$, la consideración sobre la planificación y las políticas lingüísticas, entre otras. A partir del anterior panorama, el investigador considera que existen dos propiedades centrales en la glotopolítica: la indicialidad y la normatividad. En relación con la indicialidad, afirma que el lenguaje "adquiere pleno sentido social en tanto discurso, es decir, que aparece utilizado en contextos precisos y conectados a identidades y relaciones sociales siempre en proceso de negociación” (p. 93). Es decir, la indicialidad ubica al lenguaje en un contexto espacio-temporal determinado con sujetos reales. Con respecto a la normatividad, el autor menciona que "al usar el lenguaje siempre orientamos nuestra producción verbal en función de un contexto de normas socialmente constituidas” (p. 93). En otras palabras, al usar el lenguaje, los hablantes actúan en relación con unas normas que han sido creadas y aceptadas por su comunidad de habla (por ejemplo, las formas de cortesía, de tratamiento, etc.). En este marco de comprensión, Del Valle (2007) señala que el estudio de las representaciones del lenguaje conlleva al estudio de las ideologías lingüísticas. En sus palabras:

\footnotetext{
Las ideologías lingüísticas son sistemas de ideas que articulan nociones del lenguaje, las lenguas, el habla y/o la comunicación con formaciones culturales, políticas y/o sociales específicas. Aunque permanecen en el ámbito de las ideas 200 y se pueden concebir como marcos cognitivos que ligan coherentemente el lenguaje con un orden extralingüístico, naturalizado y normalizándolo (van Dijk, 1995), también hay que señalar que se producen y reproducen en el ámbito material de las prácticas lingüísticas y metalingüisticas, de entre las cuales presentan para nosotros interés especial las que exhiben un alto grado de institucionalización. (p. 19)
}

Como muestra de la anterior concepción, Arnoux y Del Valle (2010) señalan cómo las ideologías lingüísticas están presentes en los procesos de integración o desintegración de las regiones. Estos investigadores realizan el análisis de un fragmento del discurso del presidente de Paraguay, Fernando Lugo, donde les habla a sus compatriotas, al dar a conocer sus concepciones sobre el portugués, el guaraní y el español, y cómo estas se reflejan en las políticas lingüísticas, económicas y administrativas del país. Por ejemplo, en el desarrollo de Mercosur, se perciben dos posiciones: quienes ven el portugués como un aliado o aquellos que lo ven como un enemigo con fines económicos. En el caso de Paraguay, este tratado trae retos económicos para el país que pueden ser percibidos como algo negativo. Además, en el discurso del presidente, se ve reflejado el rechazo hacia la lengua oficial de Brasil, mientras que apoya el guaraní. Otro ejemplo es el 
de NAFTA (North American Free Trade Agreement) que busca, por medio del uso del inglés, afianzar un colectivo regional. Así, para estos autores, es a partir de estas relaciones de lengua-región-comunidad que otros países toman la decisión de cuáles idiomas adoptar o no, en sus políticas lingüísticas.

Así mismo, Arnoux y Del Valle (2010) dan cuenta de cómo las ideologías lingüísticas son también el resultado de acontecimientos históricos. Citan fragmentos del siglo XIX y XX, en los que se habla de América Latina como un territorio unido y uniforme con una sola lengua que tiene dos idiomas: el español y el portugués. Esto, según los autores, buscaba reflejar una ideología latinoamericanista de unidad que se proyecta sobre el imaginario de una homogeneidad lingüística. Pero actualmente, existe una ideología orientada más hacia la parte de una comunidad iberoamericana, que incluye a España y Portugal, con lo que se hablaría de una comunidad más grande con un reconocimiento mundial que, a su vez, trae consigo el boom del idioma español. Se demuestra así, que a través de la lengua se encuentra la manera de unir la Unión Europea a Mercosur.

Derivado de la categoría de las ideologías lingüísticas, Arnoux y Del Valle (2010) desarrollan la noción de ideologema. Se usa el término “ideogema”, propuesto por Marc Angenot en 1982, para referirse a postulados o máximas que funcionan como presupuestos del discurso y que se pueden identificar en diferentes épocas, instituciones o campos discursivos. Se afirma que se establece un ideologema cuando se logra naturalizar, es decir, cuando logra ser aceptado por la sociedad. En general, se recurre a diversas apreciaciones emocionales como: la lengua es patria común o el español es lengua universal. Sin embargo, los ideologemas no se reducen a frases o sintagmas; por el contrario, para Del Valle (2005 citado en Luria, 2009, p. 3), los ideologemas se asocian con la doxa en tanto constituyen opiniones e ideas consagradas y evidencias comunes aceptadas por la mayoría, que no se someten a discusión. En otras palabras, los ideologemas son lugares comunes que integran sistemas ideológicos más abarcadores.

Otra categoría que se suma en la comprensión de las ideologías lingüísticas está relacionada con la construcción de identidades. Para, Arnoux y Del Valle (2010), las subjetividades y emociones ligadas al pasado siguen teniendo peso en la construcción de ideologías lingüísticas. Se dice que el español se encuentra en medio de tensiones sobre su difusión global ya que, por ejemplo, en España han existido conflictos nacionales respecto al español, el catalán, el gallego y el vasco, resultantes en una disputa nacionalista, pero a la vez se habla del español como un "lugar de encuentro".

\section{La función política de la lengua: diccionarios, gramáticas y manuales de texto}

Desde el campo glotopolítico, la función política de la lengua se ha abordado, principalmente, desde los estudios lexicográficos (diccionarios), las gramáticas, los manuales de texto y las políticas lingüísticas. Es así como se encuentran los trabajos de Lauria (2009), quien parte de la consideración de que la lengua es un elemento esencial en la configuración simbólica de la nación. En este contexto, Lauria (2009) se interesa por el estudio del diccionario de Tobías Garzón de 1910, puesto que considera que "la producción lexicográfica monolingüe se relaciona indisociablemente con la idea de nación" (p. 2). En otras palabras, aparte de reconocer y reflexionar sobre las variedades lingüísticas, en los diccionarios se configura un imaginario nacional. Así pues, para esta autora, la elaboración de un diccionario monolingüe es un acto glotopolítico, en la medida en que exige, por un lado, la toma de decisiones en torno a asuntos como la norma, el uso, la prescripción y descripción, el cambio lingüístico, la corrupción idiomática, los barbarismos, calcos, préstamos, entre otros; por otro lado, evidencia "fenómenos que pertenecen al ámbito político de la coyuntura histórica en la que se inserta" (p. 2).

En este orden de ideas, en el análisis del diccionario referenciado, Lauria situó el contexto en el cual se originó: el Centenario de la Revolución de Mayo, periodo de gran importancia ya que "representa por un lado la etapa de consolidación del proyecto nación y; por otro, un movimiento migratorio que trae como 
consecuencia una vasta variedad lingüística social y cultural en el país" (p. 3). De allí que la autora reflexione sobre asuntos pertenecientes a la nación, la nacionalidad y el tema de identidad, sobre dichas variedades.

En cuanto a la identidad lingüística, "esta es objeto de estudio en la medida en que representa uno de los componentes de la identidad nacional" (Lauria, 2009, p. 4). Para su análisis, toma representaciones con respecto a la lengua o idioma nacional, puesto que "la construcción de las mismas sugiere un aspecto conflictivo y dinámico, es decir, que la instauración de representaciones se cierra cuando se logra el consenso, cuando estas son incorporadas en tanto prácticas" (p. 5); adicionalmente, "las representaciones se vinculan con posicionamientos lingüísticos-políticos-ideológicos que permiten interpretar su sentido histórico” (p. 6). En esta línea, la autora destaca varios fragmentos que dan cuenta de la posición que defiende Garzón. Entre ellos están los siguientes: "no hay una lengua argentina sino castellana", "extranjerismo: palabra, frase o giro exótico o ajeno a la índole del idioma castellana", "gringo: extranjero cuya lengua nativa no es la castellana [...]" (p. 6). Así, a partir de los anteriores fragmentos, subyace el tema de la unidad o de la fragmentación del castellano o del español.

De lo anterior se desprende otro aspecto relacionado con el léxico como única marca de identidad lingüística nacional. Lauria (2009) señala que "cada una de las comunidades nacionales hispánicas ha desarrollado un léxico diferente del de las otras en muchos campos relacionados con la civilización moderna, como manifestación de sus propias historias" (p. 10); así pues, en el léxico "concluyen aportes de diversos orígenes: lo antiguo y lo nuevo; lo nacional y lo extranjero; lo indígena y lo hispánico” (p. 11). En definitiva, para Lauria, la producción lexicográfica está intrínsecamente asociada con la idea de nación ${ }^{2}$. "En el diccionario se pone de manifiesto la preservación de la unidad de la lengua española o castellana, se muestra un criterio contrastivo con respecto al DRAE y se registra argentinismos como signo característico de la identidad lingüística” (p. 11). Como resultado, dos de las ideologías lingüísticas, las cuales atienden a una pertenencia a un sistema determinado, son: la cultura monoglósica y la prohispanista, las cuales se desprenden de las representaciones ${ }^{3}$ de la lengua nacional.

Ahora bien, adicionalmente al interés del estudio de los diccionarios, también surgen investigaciones donde las gramáticas se configuran en el eje central de reflexión glotopolítica. Arnoux (2008a) señala que:

La exposición del saber sobre las lenguas, tal como aparece en las gramáticas, en la medida en que asigna legitimidad a las formas y determina el patrón al que deben ajustarse las prácticas, interviene como una dimensión de la política conformando un imaginario de la lengua e instaurando un dispositivo disciplinador que permita estructurar y estabilizar el universo social. (p. 201)

En este sentido, las gramáticas ${ }^{4}$ son productos de procesos político-sociales que se instauran en los sistemas educativos y que implican procesos de larga duración, como se dio en la consolidación de los Estados (caso de Chile). Como lo indica Arnoux, en la consolidación de los Estados nacionales se afirma la "cultura lingüística monoglósica" que, para Del Valle (2005 citado por Arnoux, 2008b), implica la articulación de dos principios: el primero de ellos tiene que ver con la focalización, el cual "refleja la idea de que hablar es siempre usar una gramática, entendida como sistema bien definido y mínimamente variable” (p. 205). El segundo se relaciona con la convergencia, la cual "presupone que el comportamiento verbal de los miembros de una comunidad tiende a hacerse más y más homogéneo con el paso del tiempo” (Del Valle, 2014, p. 30). Así, la gramática ha ejercido un rol determinante en la configuración de una representación de lengua construida históricamente en la cultura monoglósica. Por ello, Arnoux (2008a, p. 206) se interesa en cómo "la gramática construye representaciones de lo social desde su discurso acerca de la lengua" en el plano diacrónico, en la medida en que la glotopolítica indaga las formas de articulación con procesos históricos de larga duración, es decir, adquiere relevancia el estudio de los trayectos históricos amplios para comprender la red de gramáticas ${ }^{5}$.

Además del estudio de los diccionarios y de las gramáticas, la investigación de la función política de la lengua también se ha centrado en el análisis de los manuales normativos, ya que estos son huellas del discurso del idioma nacional. Para Zaccari (2010), 
La lengua es pensada, por un lado, como elemento constituyente de la identidad nacional ("La lengua de la nación”) y, por otro, como elemento cohesivo que facilita la comunicación entre los ciudadanos y, por ende, la búsqueda de un consenso político ("el interés nacional"). Ser nacional y hablar la "lengua de la nación" son, en esta causa, la misma cosa. (p. 383)

Así, en este contexto, la lengua es un elemento fundamental de la nacionalidad, puesto que garantiza una filiación y lealtad al Estado.

Una huella, del proyecto de los Estados, sobre el "idioma nacional" se ve plasmado en las políticas y planificación lingüística, específicamente en los manuales escolares. Así:

A comienzos de los años 90, surgen trabajos del equipo dirigido por Elvira Arnoux que estudia las herramientas escolares como discursos de intersección entre las tradiciones pedagógicas y disciplinares, y las formaciones sociales ideológicas. El conjunto de estos trabajos contribuyó a mostrar el modo en que la planificación lingüístico-pedagógica se planteó como estrategia para homogeneizar y disciplinar a los nuevos grupos de acceso a la vida política. Estas investigaciones son referente ineludible en el trabajo con instrumentos de gramaticalización como espacios de lucha por el poder simbólico de la nación. (López, 2016, p. 59)

En efecto, la necesidad de la homogeneización del "idioma nacional" fue un proyecto que se dio en dos sentidos: interno, en la medida en que la homogeneización permitió civilizar al pueblo y, externo, porque esta permitiría la construcción de Estados nacionales.

\section{Las políticas lingüísticas: normatividad y regulación lingüísticas}

Estudios actuales sobre el lenguaje se han interesado por el marco social, político y cultural que engloba, sitúa y evidencia los diferentes roles que el lenguaje cumple en contextos determinados. En el caso de la glotopolítica, esta atiende "a cómo los hablantes gestionan las diferencias lingüísticas y se posicionan respecto de ellas" (Arnoux y Nothstein, 2011, p. 38), y también se interesa por asuntos como las ideologías, las políticas y la planificación lingüística, sin perder de vista aquellos elementos sociales que las originan o que indicen en ellas.

Por lo anterior, Arnoux (2008b) se preocupa sobre la incidencia de las transformaciones económicas, sociales, políticas y tecnológicas en el campo glotopolítico. De allí que la autora parta de la definición de globalización, propuesta por Habermas (2000 citado por Arnoux, 2008b, p. 1), la cual hace referencia a un proceso y no a un estado, en el que el aspecto más importante lo constituye la globalización económica: la extensión e intensificación del comercio de bienes industriales entre Estados.

Para Arnoux (2008b), algunos de los problemas o intervenciones en el espacio del lenguaje son: la libre circulación de bienes culturales y lingüisticos, en la que se propone replantear una política lingüística que logre establecer un equilibrio entre la libertad de circulación de servicios y la protección de la lengua propia; la conformación de grandes conglomerados empresariales, al tener en cuenta el número de individuos, empresas y convenios internacionales que han propendido por políticas lingüísticas proteccionistas que impulsen el reconocimiento de las lenguas de trabajo y las lenguas globales; la multiplicación de organismos internacionales, la cual "plantea el tema de las lenguas oficiales y de trabajo en determinados lugares" ${ }^{6}$; la internalización de los avances cientificos, al causar la expansión de un lengua y el desarrollo tecnológico y terminología, ya que:

El desarrollo tecnológico impulsa la reflexión en el campo, que en su avance, compromete más a las áreas lingüísticas. En este caso, estas últimas o dejan que las palabras se adopten sin opciones controladas en la propia lengua o comienzan a considerar necesario el trabajo terminológico permanente para mantener una lengua actualizada para los usos especializados. (Arnoux, 2008 b, p. 4)

En definitiva, se requiere articular las ciencias sociales con un saber lingüístico especializado.

Así mismo, otras de las intervenciones o problemas planteados por Arnoux (2008b) tienen relación con: la expansión de internet; el inglés como lengua mundial, por ser la más posicionada para intercambios comunicativos; el uso de otras lenguas globales, que han aspirado a una posición más alta o "globalizada"; la 
importancia de las áreas lingüísticas, ya que estas gozan de una posición económica privilegiada y su lengua les permite avanzar económicamente sobre zonas y países que la comparten; la visibilidad de la diversidad interior, en la que es un hecho significativo haber descubierto las minorías lingüísticas de su territorio, oficializar lenguas que antes no eran reconocidas e implementar una legislación con respecto a la diversidad lingüística; las integraciones regionales, que al ser necesarias para el desarrollo económico, deben asentarse en un espacio articulado políticamente que desarrolle prácticas de participación, todo lo cual ha llevado a reflexionar sobre el papel de las lenguas y la promoción del aprendizaje de lenguas de la zona integrada.

En esta línea, Arnoux (2008b) señala que, en el marco de la política lingüística ${ }^{7}$ y la glotopolítica, los materiales normativos se configuran en elementos de gran valor de estudio, porque se toman como:

[...] objetos de reflexión glotopolíticos por su circulación en ámbitos públicos, su incidencia en las ideologías, su participación en debates que han marcado una época y sostienen decisiones que afectan el espacio del lenguaje o la reproducción de las diferencias sociales. Enfocarlos glotopolíticamente implica analizarlos en relación con procesos históricos, cambios en las tecnologías de la palabra, nuevas relaciones entre clases dirigentes y los sectores populares, necesidades de mercado de trabajo o transformaciones en los sistemas económicos. (Arnoux y Bein, 2010, p. 338)

Con todo lo anterior, es importante reconocer que las consecuencias o problemas que afectan las sociedades, derivados de fenómenos lingüísticos, harán alusión a procesos de cambios continuos.

Así, para Arnoux (2008b), las políticas lingüísticas cumplen varias funciones, entre ellas están: expresar la oficialidad de las lenguas en los Estados, determinar planes y acciones en los sistemas educativos, administrativos y otros medios y estimular y orientar procesos de integración en los que la enseñanza de lenguas cumple un papel fundamental. Los trabajos de Arnoux (Arnoux, 2008a, 2008b, 2014, 2016; Arnoux y Bein, 2010, 2015; Arnoux y Del Valle, 2010; Arnoux y Nothstein, 2011) demuestran un estudio de la evolución de la política lingüística en los años 80 y 90 . Al respecto la autora señala que:

Las políticas lingüísticas se pensaban fundamentalmente en marcos nacionales, atendiendo, por ejemplo, a las lenguas que se debían enseñar en el sistema educativo, a la integración de los hablantes de lenguas minoritarias, a las designaciones de los espacios públicos, a la regulación de la publicidad a las lenguas que podían circular en los medios. A partir de los años 90, la expansión de los nuevos sistemas de comunicación y la puesta en red a escala mundial de individuos e instituciones y la constitución de mercados globales así como el crecimiento de los traslados, los movimientos migratorios y los múltiples acuerdos internacionales, replantearon el alcance y los términos de las políticas lingüísticas. (Arnoux 2006 citada por Ardini y Moyano, 2015, pp. 180-181)

De hecho, en el contexto latinoamericano, Arnoux (2014) se ha ocupado del tema de las políticas lingüísticas e integración regional sudamericana, puesto que dicha integración se caracteriza por ser "un proceso en marcha para lo cual lo cultural y lo político son instrumentos importantes en la lucha por su conformación definitiva" (Unasur, 2008 citado por Arnoux y Bein, 2010, p. 12). En este sentido, la unión que se pretende establecer, requiere de la participación ciudadana y su formación en valores y principios, como también de "políticas lingüísticas que permitan conocer y vincularse con el otro" (p. 13). Así, para Arnoux (2014), construir una identidad política y ciudadanía sudamericanas y desarrollar un espacio regional integrado en lo político, económico, social, cultural, ambiental, energético y de infraestructura, contribuye al fortalecimiento de la unidad de América Latina y el Caribe.

\section{Reflexión final}

La revisión de las investigaciones adelantadas en el campo de la glotopolítica refleja el creciente interés por estudiar el lenguaje articulado con la política. En el contexto latinoamericano, especialmente desde Argentina, se han realizado estudios que nos permiten comprender cómo a través de la producción de manuales y libros de texto se activan representaciones ideológicas del lenguaje que se ponen en juego en el ámbito escolar. Así mismo, estos desarrollos han aportado al estudio de las políticas lingüísticas, especialmente, en países donde 
se han firmado tratados de comercio. En este marco de reflexión, las preguntas de qué se regula, quién regula, cómo y cuándo se regula hacen parte de la planificación lingüística, la cual hoy en día no solo esta mediada por asuntos puramente lingüísticos sino también por aspectos políticos y económicos. De allí que, algunas de las temáticas derivadas para futuras investigaciones giren en torno al panhispanismo como un dispositivo de homogeneidad lingüística, el valor económico del español, entre otras.

\section{Referencias}

Ardini, C., y Moyano, L. F. (2015). Políticas del lenguaje, integración regional y legislación. Posibilidades de articulación. En E. Arnoux y R. Bein (eds.), Politica lingüistica y enseñanza de lenguas (pp. 175-194). Buenos Aires: Editorial Biblos.

Arnoux, E. N. de (2008a). Los discursos sobre la nación y el lenguaje en la formación del Estado (Chile, 1842-1862). Estudio glotopolítico. Buenos Aires: Santiago Arcos.

Arnoux, E. N. de (2008b). Ámbitos para el español: recorridos desde una perspectiva glotopolítica. REVERTE. Revista de Estudos e Reflexões Tecnológicas da Faculdade de Indaiatuba, 6, 1-28. Recuperado de http://reverte.fatecid.c om.br/index.php/revista/article/view/25/29

Arnoux, E. N. de (2014). Glotopolítica: delimitación del campo y discusiones actuales con particular referencia a Sudamérica. En L. Zajícová y R. Zámec (eds.), Lengua y politica en América Latina: perspectivas actuales. Actas del II Coloquio Internacional de Estudios Latinoamericanos de Olomouc. Olomouc, CZ: Univerzita Palackého v Olomouci [12 de marzo de 2017, Artículo en un blog]. Recuperado de http://panhispanica.blogspot.com.co /2017/03/glotopolitica-delimitacion-del-campo-y.html

Arnoux, E. N. de (2016). Minorización lingüística y diversidad: en torno al español y al portugués como lenguas científicas. En Ministério da Cultura do Brasil \& Instituto de Patrimônio Histórico e Artístico Nacional (eds.), Anais 5. Seminário Ibero-americano da Diversidade Linguistica. 17 a 20 de novembro de 2014. Foz do Iguaçu, Paraná (pp. 290-306). Brasilia: Instituto de Patrimônio Histórico e Artístico Nacional. Recuperado de http:// dspace.unila.edu.br/123456789/3616

Arnoux, E. N. de, y Bein, R. (2015). Política lingüistica y enseñanza de lenguas. Buenos Aires: Editorial Biblos.

Arnoux, E. N. de, y Bein, R. (comps.). (2010). La regulación política de las prácticas lingüisticas. Buenos Aires: Eudeba.

Arnoux, E. N. de, y Del Valle, J. (2010). Las representaciones ideológicas del lenguaje: discurso glotopolítico y panhispánico. Spanish in Context, 7(1), 1-24. https://doi.org/10.1075/sic.7.1.01nar

Arnoux, E. N. de, y Nothstein, S. (2011). Desde Iguazú: mirada glotopolítica sobre la integración regional. En A. Fanjul y G. Da Silva Castela (orgs.), Linguas, Politicas e Ensino na Integração Regional (pp. 38-64). Cascavel, BR: Editora Assoeste.

Del Valle, J. (2007). La lengua, ¿patria común? Ideas e ideologias del español. Madrid: Iberoamericana.

Del Valle, J. (2014). Lo político del lenguaje y los límites de la política lingüística panhispánica. Boletín de Filología, 49(2), 87-112. Recuperado de https://boletinfilologia.uchile.cl/index.php/BDF/article/view/35824/37470

Lauria, D. (2009, abril). Los discursos sobre la lengua (1900-1910). Un abordaje desde la teoría del discurso social. Trabajo presentado en el IV Coloquio de Investigadores en Estudios del Discurso y I Jornadas Internacionales sobre Discurso e Interdisciplina, Córdoba, Argentina. Recuperado de http://www.fl.unc.edu.ar/aledar/index.php?op tion=comwrapper\&Itemid $=47$

López, M. (2016). Nosotros, vosotros, ellos. La variedad rioplatense en los manuales escolares. Buenos Aires: Miño Dávila Editores.

Zaccari, V. (2010). Sobre la regulación de la ciudadanía. Análisis del objeto discursivo “idioma nacional” en textos normativos. En E. N. de Arnoux y R. Bein (comps.), La regulación política de las prácticas lingüisticas (pp. 361-394). Buenos Aires: Eudeba. 


\section{Notas}

* Artículo de investigación científica El artículo que se presenta es producto de los avances desarrollados en la tesis doctoral titulada Ideologemas sobre los procesos de formación de excombatientes después de la firma del Acuerdo de Paz en Colombia, que hace parte del "Énfasis de Lenguaje y Educación", en la línea de investigación Narraciones, argumentaciones, justificaciones y discurso en la formación ética y política, bajo la dirección de la Dra. Marieta Quintero Mejía (PhD), desarrollada en el Doctorado Interinstitucional en Educación de la Universidad Distrital Francisco José de Caldas.

1 Para Joseph (2006), la glotopolítica se interesa por la negociación de "cualquier situación en la que haya una distribución desigual de poder" (citado por Del Valle, 2014, p. 91).

2 "El discurso lexicográfico propone una determinada representación de la sociedad y forja un determinado imaginario nacional que se aspira a instaurar". En este punto, los diccionarios "operan como instrumentos al servicio de la construcción de la nación (Lauria, 2010, p. 731).

3 "Las representaciones materializadas en el instrumento lingüístico seleccionado en torno a la lengua nacional desarrollan un concepto de lengua que enuncia una finalidad identitaria de unidad nacional o de vínculo de nacionalidad [...] los diccionarios producen y reproducen dichas representaciones debido al valor simbólico de autoridad de la lengua que ostentan determinadas ideologías lingüísticas" (Lauria, 2009, p. 12). De las representaciones de la lengua nacional, se desprenden dos identidades lingüísticas: por un lado, la cultura monoglósica, acorde con Vázquez Villanueva (2006 citado en Lauria, 2009, p. 13), "expande el deseo de borramiento de la diversidad lingüística, pretende concretar la imposición de una lengua sobre otras y circunscribe el marco hegemónico de una sociedad fuertemente homogénea y regida por la uniformidad", mientras que la prohispanista reconoce que hay un legado español.

$4 \quad$ Para López (2016), la gramática escolar se configura en un acto glotopolítico, en la medida en que supone decisiones sobre la variedad a enseñar y la teoría didáctica a implementar.

5 Las gramáticas generales han sido producto del carácter normativo de la lengua, pero, la aparición de las gramáticas particulares da cuenta de la heterogeneidad de las prácticas lingüísticas, y con ello se configuran en formas de gramáticas nacionales.

6 En este tema, Arnoux señala que la "minorización de los intercambios verbales por falta de dominio de la(s) lengua(s) dominantes en cada ámbito incide en la toma de decisiones y subordina a los hablantes de otra lengua” (2008b, p. 3).

7 En cuanto a la planificación lingüística, Arnoux (2008b) la comprende como el estudio de las acciones sobre la lengua en dos esferas. La primera está relacionada con el corpus: fijación de una escritura, elaboración de glosarios, control de préstamos, estandarización, etc. y la segunda, el estatus: promoción de una lengua subyugada a la posición de lengua nacional o global, oficialización de lenguas minoritarias, etc. (p. 2).

\section{Licencia Creative Commons CC BY 4.0}

Cómo citar este artículo: Molina Ríos, J. A. (2019). Estudios del lenguaje desde una perspectiva glotopolítica. Signo y Pensamiento, 38(74). https://doi.org/10.11144/Javeriana.syp38-74.elpg 